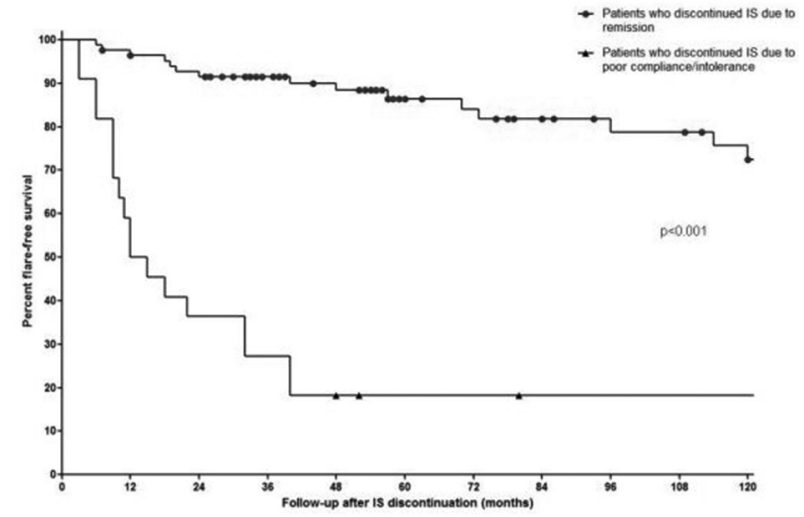

Abstract 173 Figure 1 Flare-free survival in patient who discontinued IS due to remission or due to poor compliance/intolerance along the follow-up after IS discontinuation

enable IS withdrawal, but no enough evidence is available regarding the benefits of IS discontinuation, especially in nonrenal SLE, or the risk of a subsequent flare. Our aims were to assess the rate of IS withdrawal in SLE remitted patients and the predictors of a subsequent flare and flare-free survival; moreover, to evaluate the effect of IS withdrawal on damage accrual. Methods We studied patients diagnosed with SLE between 1990 and 2018 treated with IS over their disease course who discontinued ISs. IS discontinuation was defined as complete withdrawal of any IS. Reasons for discontinuation were: remission, defined as clinical SLE Disease Activity Index (SLEDAI)$2 \mathrm{~K}=0$, or poor compliance/intolerance. Flares were defined according to SLEDAI Flare Index. Predictors of a subsequent flare and flare-free survival were analyzed by multivariate logistic regression and Cox regression analysis, respectively.

Results Eligible patients ever treated with IS were 319 out of 456. 139 patients discontinued ISs, 105 of them (75.5\%) due to remission. Mean \pm SD follow-up after IS withdrawal was 91 \pm 71 months (range 6-372).

26/105 remitted (24.7\%) and 23/34 unremitted patients $(67.6 \%)$ experienced a flare $(\mathrm{p}<0.001)$, a median (range) of 57 (6-264) and 8 months (1-72) after IS discontinuation, respectively $(p=0.009)$. Flare-free survival is depicted in figure 1 .

Table 1 reports characteristics of remitted patients, overall and according to flare occurrence after IS discontinuation. At multivariate logistic regression analysis, antimalarial intake (OR, 95\% CI 0.103, 0.023-0.452, $\mathrm{p}=0.003$ ), older age (OR 0.943, 0.892-0.997, $\mathrm{p}=0.039$ ) and the duration of remission before IS discontinuation (OR 0.943, 0.938-0.996, $\mathrm{p}=0.025$ ) were independent protective factors against flares after IS discontinuation. Conversely, no predictive factors of a longer time-to-flare were identified by multivariate Cox-regression analysis.

Median damage accrual was similar in patients who discontinued (1, range $0-8)$ or not IS (1, range $0-9)$ after adjusting for age, disease duration and cumulative prednisone dose.

We separately analyzed data of non-renal remitted patients (37 patients, $35.2 \%)$. The only variable associated with a lower flare rate was antimalarial intake (OR 0.048, 0.005-0.503, $\mathrm{p}=0.002$ ).

Conclusions In our cohort, one third of patients treated with IS discontinued the drug during the follow-up, in most cases due to remission. The use of antimalarials after IS discontinuation was independently associated with a significant decrease in the risk of flare.
Funding Source(s): None

Table 1 reports characteristics of remitted patients, overall and according to flare occurrence after IS discontinuation.

\section{SLE RESPONDER INDEX (SRI) UNDERESTIMATES CLINICAL RESPONSE IN MUSCULOSKELETAL SYSTEMIC LUPUS ERYTHEMATOSUS}

${ }^{1}$ Khaled Mahmoud, ${ }^{2}$ Ahmed Zayat, ${ }^{1}$ Md Yuzaiful Md Yusof, 'Elizabeth Hensor, 'Philip Conaghan, 'Paul Emery, 'Edward Vital*. 'University of Leeds; ${ }^{2}$ Bradford Teaching Hospitals NHS Foundation Trust

\subsection{6/lupus-2019-Ism.174}

Background Musuloskeletal (MSK) manifestations are common in SLE. Many recent clinical trials were negative or had small benefits vs. placebo. SRI is a common primary endpoint but has not been independently validated. Ultrasound is an objective measure of synovitis validated in inflammatory arthritis. We previously reported that in patients with inflammatory symptoms, 38\% had swollen joints, 27\% had subclinical inflammation (abnormal US but no clinically swollen joints) and $35 \%$ had no clinical or US inflammation. We also showed that subclinical tenosynovitis and PD were associated with significantly higher IgG, physician visual analogue score, tender joint count (Zayat et al. Rheumatology 2018). The purpose of the present study was to use ultrasound as a gold standard to evaluate clinical outcome measures of MSK lupus.

Methods A prospective pilot study was conducted in consecutive SLE patients with inflammatory musculoskeletal symptoms. Clinical assessments including SLEDAI-2K, BILAG-2004, 28-tender and swollen joint counts, physician and patient VAS and ultrasound were performed at 0,2 and 4 weeks following $120 \mathrm{mg}$ intramuscular methylprednisolone acetate. Responsiveness was analysed using changes and effect sizes using Cohens criteria.

Results 20 patients were recruited. 15/20 had clinical swelling at baseline. The others had abnormal ultrasound and/or early morning stiffness and tenderness. All clinical and US parameters were significantly improved at week 4 (all p0.01). Musculoskeletal-BILAG score improved in 16/20. MusculoskeletalSLEDAI improved in $7 / 20$. SRI-4 criteria were assessed in 19 patients with SLEDAI $\geq 4$ at baseline met in $9 / 19$ at 4 weeks. Effect sizes at 4 weeks were large $(>0.5)$ for US (power Doppler and Greyscale), physician VAS and BILAG and medium $(>0.3)$ for joint counts and SLEDAI. Large effect sizes for improvement in US greyscale and power Doppler were observed in both SRI responders $(\mathrm{r}=-0.51$ and -0.56 respectively) and non-responders $(\mathrm{r}=-0.62$ and -0.59$)$ at 4 weeks. In SRI non-responders swollen joint counts improved by $20 \%$, $50 \%$ and $70 \%$ in $7 / 10,7 / 10$ and $6 / 10$ patients respectively.

Conclusions This is the first study to measure the responsiveness of clinical outcome measures in musculoskeletal SLE against an objective inflammation measure. BILAG-2004 and physician VAS were the most responsive clinical instruments. US was highly responsive in musculoskeletal SLE, while SLEDAI-2K and joint counts appeared suboptimal for detection of improvement. These results suggest that clinical trials based on the SLEDAI-2K and SRI-4 may underestimate the efficacy of therapy in SLE.

Funding Source(s): LupusUK, NIHR 


\section{Tender Joint Count}

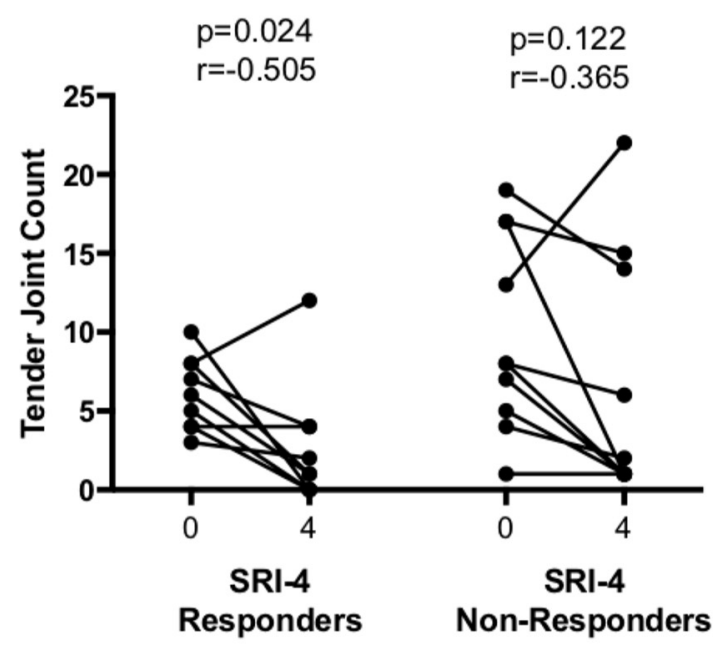

Total GS Score

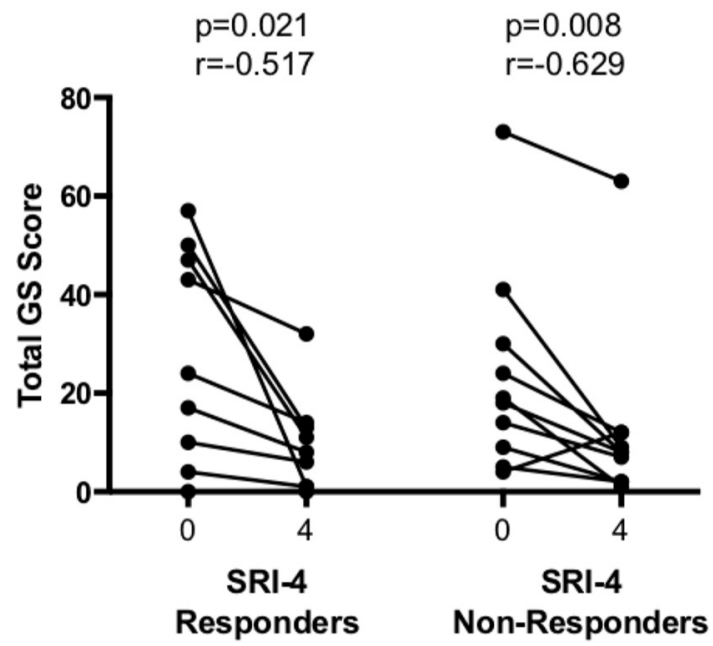

Abstract 174 Figure 1

\section{LOW DISEASE ACTIVITY STATE (LDAS) PREDICTS A BETTER HEALTH-RELATED QUALITY OF LIFE (HRQOL) IN SYSTEMIC LUPUS ERYTHEMATOSUS (SLE) PATIENTS}

${ }^{1}$ Manuel F Ugarte-Gil ${ }^{*},{ }^{2}$ Rocío Gamboa-Cárdenas, ${ }^{3}$ Cristina Reátegui-Sokolova, ${ }^{2}$ Mariela Medina-Chinchón, ${ }^{2}$ Francisco Zevallos, ${ }^{1}$ Claudia Elera-Fitzcarrald, ${ }^{2}$ Victor Pimentel-

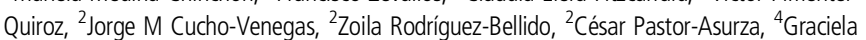
S Alarcón, ${ }^{2}$ Risto Perich-Campos. 'Universidad Científica del Sur; ${ }^{2}$ Hospital Guillermo Almenara Irigoyen. EsSalud; ${ }^{3}$ Unidad de Investigación para la Generación y Síntesis de Evidencias en Salud. Universidad San Ignacio de Loyola; ${ }^{4}$ School of Medicine. The University of Alabama at Birmingham

\subsection{6/lupus-2019-Ism.175}

Background LDAS is an achievable goal in the treatment of SLE patients and it is associated with a lower probability of flares, damage and mortality; whether HRQoL is also

\section{Swollen Joint Count}

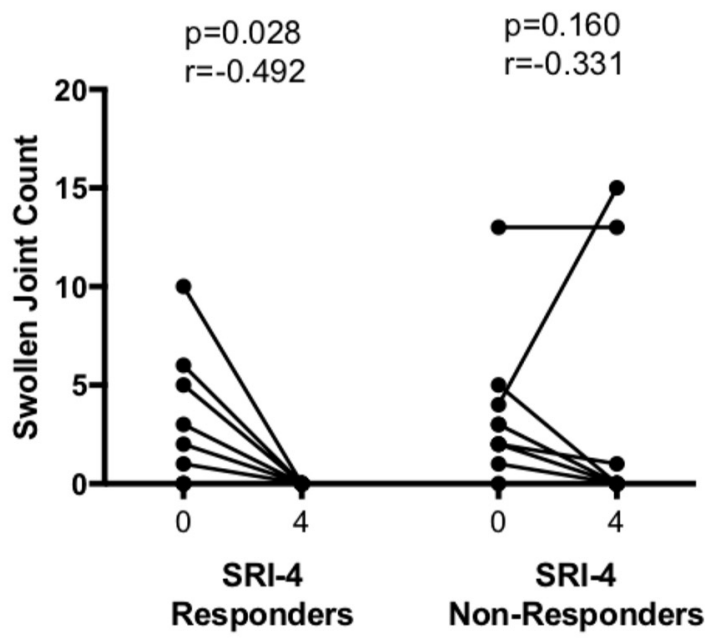

Total PD Score

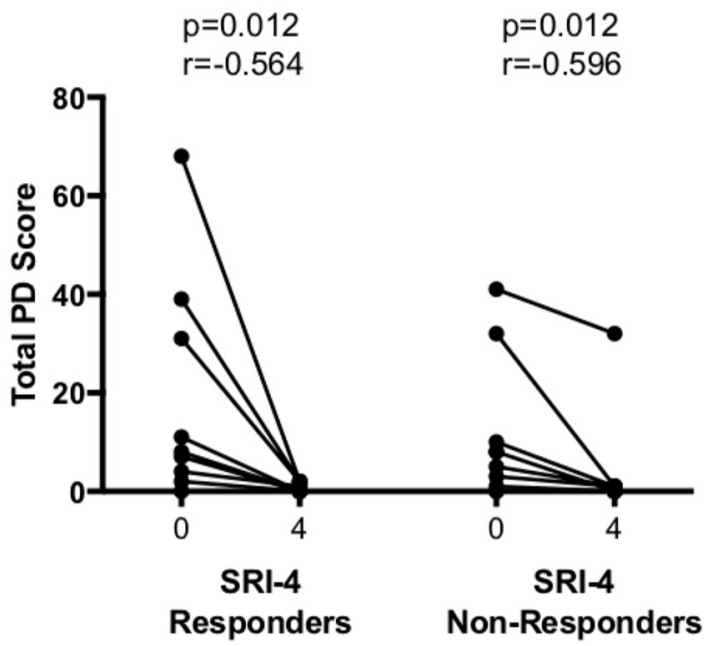

influenced hy LDAS has only been evaluated cross-sectionally but not longitudinally. The aim of this study was to determine if achieving LDAS predicts a better HRQoL

Methods SLE patients from a single center cohort with at least two visits were included. Visits were performed every six months. HRQoL was measured with the LupusQoL, disease activity with the SLEDAI-2K, damage with the SLICC/ACR damage index (SDI) and comorbidities with the Charlson Comorbidity Index (CCI). LDAS was defined as a SLEDAI-2K4, prednisone daily dose $7.5 \mathrm{mg} / \mathrm{d}$ and immunosuppressive drugs on maintenance dose; patient on remission were also included in this group. Generalized estimating equations were performed, using as outcome each one of the eight components of the LupusQoL in the subsequent visit, and the activity state in the previous visit; as possible confounders, we included disease duration, SDI, CCI, 